PROCEEDINGS OF THE

AMERICAN MATHEMATICAL SOCIETY

Volume 129, Number 4, Pages 1123-1126

S 0002-9939(00)05657-4

Article electronically published on October 16, 2000

\title{
DIVERGENT LAGUERRE SERIES
}

\author{
KRZYSZTOF STEMPAK \\ (Communicated by Christopher D. Sogge)
}

\begin{abstract}
We prove failure of a.e. convergence of partial sums of Laguerre expansions of $L^{p}$ functions for $p>4$. The idea which is used goes back to Stanton and Tomas. We follow Meaney's paper (1983), where divergence results were proved in the Jacobi polynomial case.
\end{abstract}

\section{INTRODUCTION}

Let $L_{n}^{a}(x)$ denote the $n$th Laguerre polynomial of order $a>-1$ [Sz, p. 101]. For any $p, 1 \leq p \leq \infty$, and $h$ in $L^{p}\left(x^{a} e^{-x} d x\right)$ (all Lebesgue spaces we consider live on $(0, \infty)$ and $d x$ denotes the Lebesgue measure there) we associate to $h$ its Laguerre polynomial series

$$
\sum_{0}^{\infty} b_{n} L_{n}^{a}(x)
$$

provided the coefficients

$$
b_{n}=\frac{\Gamma(n+1)}{\Gamma(n+a+1)} \int_{0}^{\infty} h(x) L_{n}^{a}(x) x^{a} e^{-x} d x
$$

exist (this is the case when $1<p \leq \infty$ ). It is known, [Sz p. 261(6)], that for $a>-1 / 2$ the Laguerre polynomial series of the function $x^{\mu},-(a+1)<\mu \leq$ $-(2 a+3) / 4$, is divergent for every $x>0$. In particular, $h(x)=x^{-(2 a+3) / 4}$, is an example of a function in $L^{p}\left(x^{a} e^{-x} d x\right), 1 \leq p<\frac{4(a+1)}{2 a+3}$, whose Laguerre polynomial series is divergent everywhere. In fact, more can be said. For any $a$ and $p, a>$ $-1,1 \leq p<2$, there is a function in $L^{p}\left(x^{a} e^{-x} d x\right)$ whose Laguerre polynomial series diverges everywhere. Such is the function $e^{c x}, \frac{1}{2}<c<\frac{1}{p}$ whose Laguerre polynomial series is even not summable in the Abel sense for every $x>0$. This example was furnished by Pollard [Po].

On the other hand, it is also known that for any $a>-1$ the Laguerre polynomial series of any function $f$ in $L^{2}\left(x^{a} e^{-x} d x\right)$, hence in $L^{p}\left(x^{a} e^{-x} d x\right), 2<p \leq \infty$, converges to $f$ a.e. This follows from Muckenhoupt's equiconvergence theorem [M] and the celebrated Carleson's result on a.e. convergence of partial sums of Fourier series.

Received by the editors March 19, 1999 and, in revised form, July 2, 1999.

1991 Mathematics Subject Classification. Primary 42C10; Secondary 42C99.

Key words and phrases. Laguerre polynomials and functions, divergence almost everywhere.

This research was supported in part by KBN grant \# 2 PO3A 048 and European Commission via the TMR network "Harmonic Analysis", contract no. ERB FMRX-CT970159. 


\section{THE RESULT}

We will be concerned with the a.e. convergence problem of expansions with respect to the system of Laguerre functions

$$
\mathcal{L}_{n}^{a}(x)=(\Gamma(n+1) / \Gamma(n+a+1))^{1 / 2} e^{-x / 2} x^{a / 2} L_{n}^{a}(x) .
$$

For any $p, 1 \leq p \leq \infty$, and $f$ in $L^{p}(d x)$ we associate to $f$ its Laguerre series

$$
\sum_{0}^{\infty} c_{n} \mathcal{L}_{n}^{a}(x)
$$

provided the coefficients

$$
c_{n}=\left\langle f, \mathcal{L}_{n}^{a}\right\rangle_{L^{2}(d x)}=\int_{0}^{\infty} f(x) \mathcal{L}_{n}^{a}(x) d x
$$

exist (this is the case when $a \geq 0$ and $1 \leq p \leq \infty$ or $-1<a<0$ and $(1+a / 2)^{-1}<$ $p \leq \infty)$. Muckenhoupt's equiconvergence theorem also implies a.e. convergence of partial sums of the series (2.1) for every $f$ in $L^{p}(d x)$ provided $a>-1 / 2$ and $p \in(4 / 3,4)$ or $-1<a \leq-1 / 2$ and $(1+a / 2)^{-1}<p<4$; cf. [St, Proposition 2.1]. The negative result contained in [St, Corollary 2.5] suggested the absence of a.e. convergence of partial sums for functions in $L^{p}(d x)$ for $p \in(1, \infty)$ lying outside the interval $(4 / 3,4)$ in the case $a>-1 / 2$. In fact, the function $f(x)=h(x) e^{-x / 2} x^{a / 2}$, $h(x)=x^{-(2 a+3) / 4}, a>-1 / 2$, is in $L^{p}(d x), 1 \leq p<4 / 3$, and its Laguerre series (2.1) diverges for every $x>0$.

Our result almost clarifies the situation on the right of the interval $(4 / 3,4)$.

Theorem 2.1. Assume $a>-1$ and $4<p \leq \infty$. There is a function $f$ in $L^{p}(d x)$ such that its Laguerre series (2.1) diverges for almost every $x>0$.

The proof of Theorem 2.1 is based on precise asymptotics of the Laguerre functions $\mathcal{L}_{n}^{a}(x)$.

Lemma 2.2 ( $\mathrm{Ma}$ Lemma 1]). Let $a \geq 0$ and $1 \leq p \leq \infty$ or $-1<a<0$ and $1 \leq p<-2 / a$. Then

$$
\left\|\mathcal{L}_{n}^{a}\right\|_{p} \sim\left\{\begin{array}{lr}
n^{1 / p-1 / 2}, & 1 \leq p<4 \\
n^{-1 / 4}(\log n)^{1 / 4}, & p=4 \\
n^{-1 / p}, & 4<p \leq \infty .
\end{array}\right.
$$

Here $\tau_{n} \sim \sigma_{n}$ means that $C^{-1} \leq \tau_{n} / \sigma_{n} \leq C$ for a constant $C>0$. An immediate consequence of Hölder's inequality and (2.3) is, that for $a$ and $p$ such that $a \geq 0$ and $1 \leq p \leq \infty$ or $-1<a<0$ and $(1+a / 2)^{-1}<p \leq \infty, f$ in $L^{p}(d x)$ and $c_{n}$ 's given by (2.2) we have

$$
c_{n}=o\left(n^{3 / 4}\right)
$$

(we are slightly generous here since, in fact, there are better estimates: $c_{n}=o\left(n^{1 / 2}\right.$ ) if $1 \leq p<\infty$ and $c_{n}=O\left(n^{1 / 2}\right)$ if $\left.p=\infty\right)$. Note, that if, in addition to these assumptions on $a$ and $p$, we further restrict $p$ to $p<4$, then Hölder's inequality and (2.3) give $c_{n}=o\left(n^{1 / 4}\right)$.

We include the proof of the next lemma for the sake of completeness.

Lemma 2.3 ([M, Lemma 5]). Assume $a>-1$ and a sequence $\left\{c_{n}\right\}$ is such that $c_{n}=o\left(n^{3 / 4}\right)$ and $\lim _{n \rightarrow \infty} c_{n} \mathcal{L}_{n}^{a}(x)=0$ for all $x$ in a subset $E \subset(0, \infty)$ of positive Lebesgue measure. Then $c_{n}=o\left(n^{1 / 4}\right)$. 
Proof. By assumption, $\lim _{n \rightarrow \infty} c_{n} \mathcal{L}_{n}^{a}\left(x^{2}\right)=0$ for all $x \in E^{1 / 2}$. Given $x$ in $E^{1 / 2}$ we write Hilb's asymptotic formula, $\underline{\mathrm{Sz}}, 8.22 .4$ ], in the form

$$
\mathcal{L}_{n}^{a}\left(x^{2}\right)=J_{a}\left(2 n^{1 / 2} x\right)+O\left(n^{-3 / 4}\right)
$$

The assumption made on $c_{n}$ gives $\lim _{n \rightarrow \infty} c_{n} J_{a}\left(2 n^{1 / 2} x\right)=0$. For the Bessel function $J_{a}(t)$ we have

$$
J_{a}(t)=\left(\frac{2}{\pi t}\right)^{1 / 2} \cos \left(t+A_{a}\right)+O\left(t^{-3 / 2}\right), \quad t \geq 1,
$$

$A_{a}=-\frac{a \pi}{2}-\frac{\pi}{4}$. Hence, given $x$ in $E^{1 / 2}$ we write

$$
J_{a}\left(2 n^{1 / 2} x\right)=\pi^{-1 / 2} x^{-1 / 2} n^{-1 / 4} \cos \left(2 n^{1 / 2} x+A_{a}\right)+O\left(n^{-3 / 4}\right),
$$

and again, by using the hypothesis made on $c_{n}$, we obtain

$$
\lim _{n \rightarrow \infty} c_{n} n^{-1 / 4} \cos \left(2 n^{1 / 2} x+A_{a}\right)=0
$$

Assuming, a contrario, that $\left|c_{n_{k}} n_{k}^{-1 / 4}\right| \geq \varepsilon$ for some $\varepsilon>0$ and an increasing subsequence $n_{k}$ we conclude, that $\lim _{n \rightarrow \infty} \cos \left(2 n_{k}^{1 / 2} x+A_{a}\right)=0$ for all $x$ in $E^{1 / 2}$, a set of positive measure. This contradicts a variant of the Cantor-Lebesgue theorem; cf. [M, Lemma 4].

Proof of Theorem 2.1. Suppose, a contrario, that for any $f$ in $L^{p}(d x), 4<p \leq \infty$, the series (2.1) converges on a set $E_{f}$ of positive measure. Then, by (2.4) and Lemma 2.3, $\left\langle f, \mathcal{L}_{n}^{a}\right\rangle_{L^{2}(d x)}=o\left(n^{1 / 4}\right)$. We will show, however, that there is a function $f$ in $L^{p}(d x)$ that does not possess this property. Consider the functionals

$$
T_{n} f=n^{-1 / 4-\delta}\left\langle f, \mathcal{L}_{n}^{a}\right\rangle_{L^{2}(d x)}
$$

on $L^{p}(d x)\left(\delta>0\right.$ is to be small enough; for instance, $\delta=\frac{1}{2}\left(\frac{1}{4}-\frac{1}{p}\right)$ will be sufficient $)$. Assume, that for every $f$ in $L^{p}(d x)$ we have $\sup _{n}\left|T_{n} f\right|<\infty$. This implies, by the Banach-Steinhaus theorem, that $\sup _{n}\left\|T_{n}\right\|<\infty$. By (2.3), this is equivalent to

$$
\sup _{n} n^{\frac{1}{4}-\frac{1}{p}-\delta}<\infty
$$

which is not possible for $\delta$ sufficiently small. Hence, there is an $f$ in $L^{p}(d x)$ such that

$$
\sup _{n} n^{-\delta} \cdot n^{-\frac{1}{4}}\left|\left\langle f, \mathcal{L}_{n}^{a}\right\rangle_{L^{2}(d x)}\right|=\infty
$$

and this contradicts the conclusion of Lemma 2.3.

Remark 2.4. We do not know what happens with a.e. convergence if $a>-1 / 2$ and $p=4 / 3$ or $p=4$ or, if $-1<a \leq-1 / 2$ and $p=4$.

\section{REFERENCES}

[Ma] Markett, C., Mean Cesàro summability of Laguerre expansions and norm estimates with shifted parameter, Anal. Math. 8 (1982), 19-37. MR 83j:40004

[Me] Meaney, C., Divergent Jacobi polynomial series, Proc. Amer. Math. Soc. 87 (1983), 459-462. MR 84c:42040

[M] Muckenhoupt, B., Equiconvergence and almost everywhere convergence of Hermite and Laguerre series, SIAM J. Math. Anal. 1 (1970), 295-321. MR 42:4948

[Po] Pollard, H., The mean convergence of orthogonal series II, Trans. Amer. Math. Soc. 63 (1948), 355-367. MR 9:426e 
[St] Stempak, K., Transplanting maximal inequalities between Laguerre and Hankel multipliers, Monats. Math. 122 (1996), 187-197. MR 97g:42010

[Sz] Szegö, G., Orthogonal Polynomials, Colloquium Publications, vol.23, American Mathematical Society, New York, 1939. MR 1:14b

Instytut Matematyczny Politechniki WrocŁawskiej, Wyb. Wyspianskiego 27, 50-370 WrocŁaW, Poland

E-mail address: stempak@im.pwr.wroc.pl 